\title{
EVALUATION OF EFFECTIVE PROPERTIES OF BASALT TEXTILE REINFORCED CERAMIC MATRIX COMPOSITES
}

\author{
Soňa Valentováa, ${ }^{a, *}$ Vladimír Hrbek $^{a, b}$, Michal Š́tejoha $^{a}$ \\ ${ }^{a}$ Czech Technical University in Prague, Faculty of Civil Engineering, Thákurova 7, 16629 Prague 6, Czech \\ Republic \\ ${ }^{b}$ Institute of Theoretical and Applied Mechanics AS CR, v.v.i., Prosecká 76, 19000 Prague 9, Czech Republic \\ * corresponding author: sona.valentova@fsv.cvut.cz
}

\begin{abstract}
The present paper is concerned with the analysis of a ceramic matrix composite, more specifically the plain weave textile fabric composite made of basalt fibers embedded into the pyrolyzed polysiloxane matrix. Attention is paid to the determination of effective elastic properties of the yarn via homogenization based on the Mori-Tanaka averaging scheme and the 1st order numerical homogenization method adopting a suitable representative computational model. The latter approach is then employed to simulate the response of the yarn when loaded beyond the elastic limits. The required mechanical properties of individual material phases are directly measured using nanoindentation with in-build scanning probe microscopy. Applicability of the proposed computational methodology is supported by the analysis of a unidirectional fibrous composite, representing the yarn, subjected to a macroscopically uniform strain.
\end{abstract}

KEYWORDS: Basalt fibers, textile composites, homogenization, periodic unit cell, damage.

\section{INTRODUCTION}

The textile reinforced composites have been gaining considerable attention in many different branches of engineering. Structural applications of these composites are now rather common owing to their specific strength, high stiffness, high temperature resistance, and relatively low cost in comparison to traditional materials. In addition, the possibility of influencing the resulting macroscopic properties during fabrication makes them very interesting. On the other hand, predicting the effective elastic and fracture properties and thus estimating the real behavior under variety of loads calls for a quite complicated computational analysis. Such an analysis grounds on the knowledge of properties of individual components, complex inner geometry depending on a technology of composite fabrication, and on a type of textile reinforcement. Mostly the carbon and glass fibers as a reinforcement are used, but attention is also accorded to the basalt fibers especially due to their good thermal stability, important in the manufactured process using high temperatures when ceramic matrices are needed in the final product. They can also substitute the carbon fibers in most applications at a fraction of price.

This paper deals with the evaluation of effective elastic properties of the basalt textile reinforced ceramic matrix composite at the level of yarns (microscale) using the Mori-Tanaka scheme and the finite element method, predicting its strength and characterizing the failure behavior. Herein, a two-dimensional plain weave textile is examined. First, experimental measurements are carried out to determine properties of individual components. Next, a suitable geometrical model based on the statistically equivalent periodic unit cell is designed and finally the nonlinear computational analysis is realized.

\section{Experimental Measurements of the Material Parameters}

A specimen of basalt fiber ceramic composite was embedded into an epoxy resin and left for 24 hours to harden in a cylindrical mold ( $25 \mathrm{~mm}$ in diameter). Approximately a $15 \mathrm{~mm}$ thick part of the sample was separated using a diamond cut-off wheel. To ensure an adequate surface roughness, the investigated sample was ground on an MD-Piano plate using 1200, 2000 and 4000 grain $\mathrm{cm}^{-2}$ grid and water as a lubricant under $5 \mathrm{~N}$ force. Consequently, a water based DPSuspension containing 3 and $1 \mu \mathrm{m}$ poly-crystalline diamonds on MD-Dur and MD-Nap cloths, respectively, was used for fine polishing. An MD-Chem cloth combined with the water-based DP-Suspension containing $0.25 \mu \mathrm{m}$ and $0.1 \mu \mathrm{m}$ diamond particles was selected for final polishing. In such a way, two surfaces for the measuring of longitudinal (Fig. 1) and transverse (Fig. 2) properties of the basalt fiber were prepared. This allowed to address a potential anisotropy of the ceramic matrix in the vicinity of the fibers. When cutting the specimen in the direction normal to the fibers resulted in the average root mean square roughness $\mathrm{R}_{q}=95.08 \mathrm{~nm}$, whereas in case of cuts along the longitudinal direction an increase of the average root mean square roughness $\mathrm{R}_{q}=300.75 \mathrm{~nm}$ was observed owing to the cleavage of fibers during preparation.

The measurements were carried out analogously for both longitudinal and transverse directions start- 


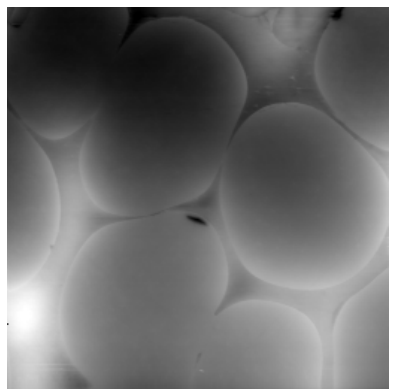

(A).

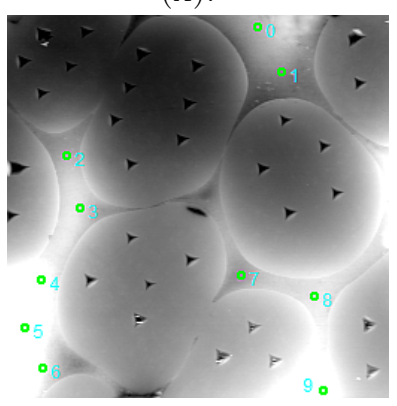

(c).

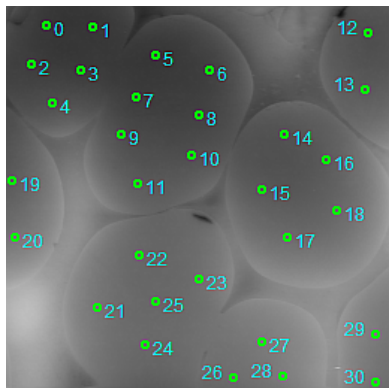

(B).

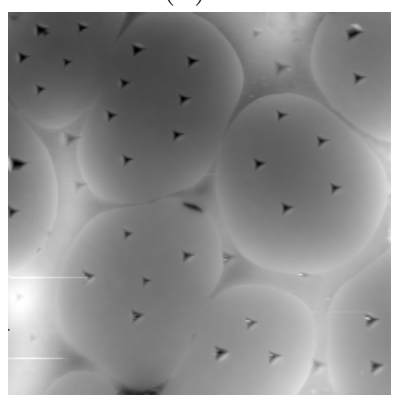

(D) .
FiguRE 1. Indentation steps in longitudinal direction.
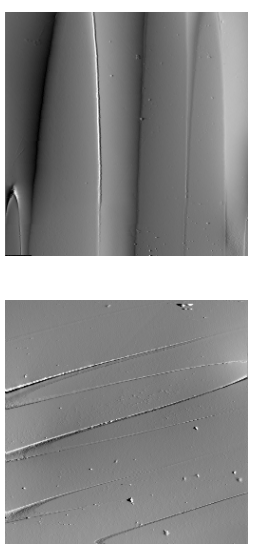

(A).
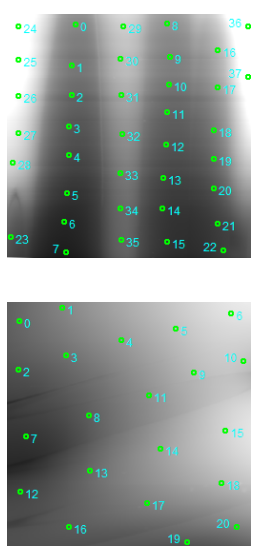

(B).
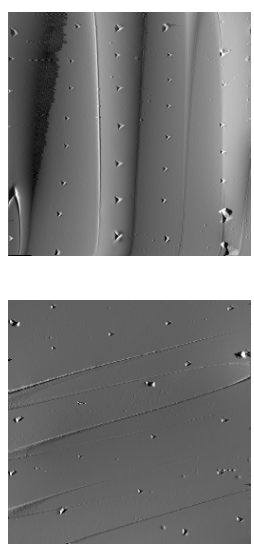

(C).
FIGURE 2. Indentation steps in transverse direction.

ing with scanning the composite surfaces with an inbuild scanning probe microscopy (SPM) (Figs. 1a and Figs. 2a) to identify location of individual indents. These are seen for the fibers in Figs. 1b and 2b. The indented surfaces are evident in Figs. $1 \mathrm{c}$ and $2 \mathrm{c}$. Each phase was subjected to a different loading function. Separation of indents ensured no interaction of individual indents. The indented surfaces were finally scanned again to check the measurements accuracy, see e.g. Fig. 1d

The load-controlled quasi-static indentation was performed with the load function consisting of "loading" and "unloading" segments lasting 5 seconds each with an in-between 300 seconds segment of "holding" time. The maximum applied force of $2500 \mu \mathrm{N}$ was used for fiber indentation and $300 \mu \mathrm{N}$ in case of the matrix. This procedure was applied to several positions on the sample. The resulting reduced moduli,

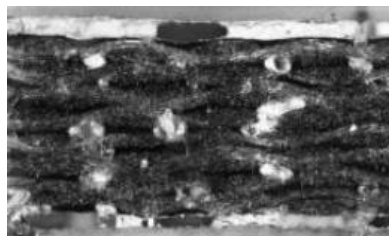

(A).

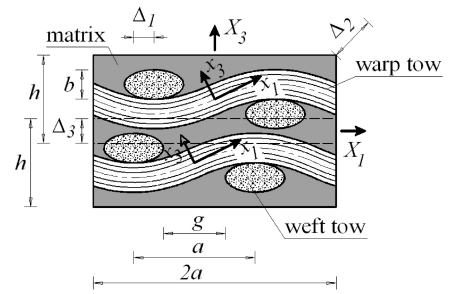

(C).

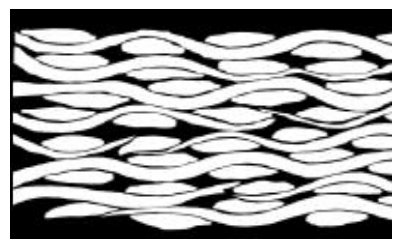

(B).

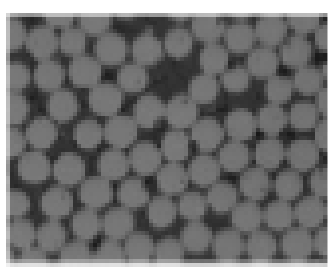

(D).
Figure 3. (A) mesoscopic 2D image of basalt fiber/ceramic matrix plain weave textile composite, (B) binary image of the 8-layer laminate cross-section, (C) description of two-layer SEPUC on the mesoscale, (D) example of a real structure of yarn.

corresponding to longitudinal and transverse indentations into the fibers and the matrix, were derived from unloading part of the indentation curve. Next, the required Young moduli were calculated based on the contact mechanics of colliding solid bodies [1, 2] assuming the Poisson ratio $\nu=0.24$ for all phases and a diamond tip with material parameters $\mathrm{E}=1140 \mathrm{GPa}$ and $\nu=0.07$. Taking into account all indents gave the average values of the Young modulus equal to 69.68 GPa and 64.82 GPa for the longitudinal and transverse fiber directions, respectively, and equal to $58.83 \mathrm{GPa}$ for the matrix. Further details including basic statistics are available in Fig. 1

It is not surprising that the fiber values are close confirming an essentially amorphous structure of the basalt fibers. A slight difference can be attributed to the quality of sample of the examined textile composite. An expected isotropy of the amorphous ceramic matrix has also been confirmed, since no principal difference has been observed when indenting the matrix in two perpendicular directions. Application of the isotropic theory of nanoindentation to both phases is therefore adequate.

\section{Formulation of Geometrical MODEL}

During construction of the geometrical model for a computational analysis of textile composite we need to distinguish several levels corresponding to its structure. Fig. 3 identifies the mesoscale structure of individual yarns and a random distribution of fibers within the yarn cross-section at the microscale level. Both scales require formulation of a suitable computational model. For illustration, a cross-section of a two-ply computational model to be used on mesoscale is plotted in Fig. 3c, see e.g. 3] for more details. 


\begin{tabular}{ccccc}
\hline Position & $\begin{array}{c}\text { Red. mod. } \\
\mathbf{E}_{r}[\mathbf{G P a}]\end{array}$ & $\begin{array}{c}\text { Young's mod. } \\
\mathbf{E}[\mathbf{G P a}]\end{array}$ & $\begin{array}{c}\text { Hardness } \\
\mathbf{H}[\mathbf{G P a}]\end{array}$ & $\begin{array}{c}\text { Contact depth } \\
\mathbf{h}_{c}[\mathbf{n m}]\end{array}$ \\
\hline Basalt fiber - longitudinal direction & & \\
\hline P127_2 & $67.81 \pm 8.09$ & $67.72 \pm 7.68$ & $5.27 \pm 0.56$ & $191.92 \pm 6.55$ \\
P128_1 & $75.95 \pm 6.55$ & $75.66 \pm 6.21$ & $5.95 \pm 0.88$ & $106.09 \pm 8.91$ \\
P128_2 & $76.85 \pm 7.75$ & $76.63 \pm 7.35$ & $5.71 \pm 0.76$ & $108.33 \pm 7.98$ \\
P130_1 & $66.17 \pm 10.44$ & $66.08 \pm 9.93$ & $5.49 \pm 0.75$ & $161.55 \pm 12.22$ \\
P130_2 & $71.76 \pm 7.30$ & $71.15 \pm 6.92$ & $5.79 \pm 0.38$ & $155.99 \pm 5.62$ \\
P130_3 & $61.12 \pm 6.68$ & $60.85 \pm 6.33$ & $5.28 \pm 0.74$ & $239.19 \pm 18.71$ \\
\hline average & $69.94 \pm 7.80$ & $69.68 \pm 7.40$ & $5.58 \pm 0.68$ & $160.51 \pm 10.00$ \\
\hline Basalt fiber - transverse direction & & \\
\hline P120_1 & $74.09 \pm 6.22$ & $73.65 \pm 6.22$ & $5.88 \pm 1.17$ & $107.60 \pm 11.80$ \\
P121_2 & $75.98 \pm 3.79$ & $75.69 \pm 3.58$ & $5.94 \pm 0.54$ & $105.58 \pm 5.57$ \\
P121_3 & $57.31 \pm 3.58$ & $56.85 \pm 3.38$ & $5.00 \pm 0.55$ & $116.33 \pm 7.25$ \\
P122_1 & $55.34 \pm 10.55$ & $54.80 \pm 10.03$ & $4.45 \pm 1.16$ & $126.64 \pm 17.08$ \\
P122_2 & $68.77 \pm 8.09$ & $68.65 \pm 7.68$ & $5.15 \pm 0.54$ & $114.39 \pm 6.80$ \\
P124_1 & $59.61 \pm 11.70$ & $59.26 \pm 9.44$ & $5.29 \pm 1.22$ & $117.95 \pm 15.13$ \\
\hline average & $65.18 \pm 7.32$ & $64.82 \pm 7.03$ & $5.29 \pm 1.19$ & $114.52 \pm 14.15$ \\
\hline Pyrolyzed polysiloxane matrix & & \\
\hline P127_2 & $67.57 \pm 3.82$ & $67.47 \pm 3.61$ & $7.29 \pm 0.96$ & $56.64 \pm 4.25$ \\
P128_1 & $56.45 \pm 3.77$ & $55.95 \pm 3.56$ & $5.44 \pm 0.44$ & $110.65 \pm 4.90$ \\
P128_2 & $60.20 \pm 5.09$ & $59.88 \pm 4.82$ & $6.01 \pm 0.54$ & $104.88 \pm 5.21$ \\
P130_1 & $56.70 \pm 8.53$ & $56.22 \pm 8.10$ & $5.63 \pm 1.02$ & $109.81 \pm 10.39$ \\
P130_2 & $58.39 \pm 7.29$ & $57.98 \pm 6.91$ & $5.68 \pm 0.99$ & $109.45 \pm 11.72$ \\
P130_3 & $55.82 \pm 5.00$ & $55.30 \pm 4.73$ & $6.23 \pm 1.08$ & $103.85 \pm 10.21$ \\
\hline average & $59.19 \pm 5.58$ & $58.83 \pm 5.29$ & $6.05 \pm 0.84$ & $99.21 \pm 7.78$ \\
\hline
\end{tabular}

TABLE 1. Indentation results of individual material phases.

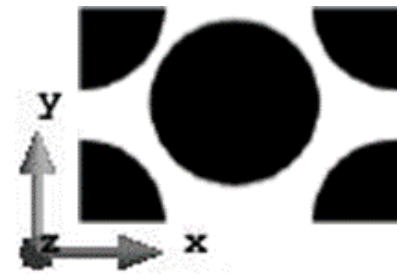

(A).

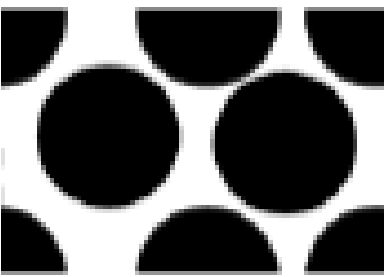

(B).
Figure 4. Microscale level of yarn, (A) two-fiber SEPUC-1, (B) four-fiber SEPUC-2.

In this paper the main attention is paid to the microscale, meaning the level of yarns. Fig. 4 plots two types of the idealized unit cells being representative to the actual yarn. These are derived by comparing material statistics of real, Fig. 3d, and artificial microstructures. Such representative volume elements (RVE) are call the statistically equivalent periodic unit cell (SEPUC), see e.g. [4] for more details. Apart from correctly capturing the real microstructure attention must also be accorded to the application of macroscopically uniform state of stress or strain and proper boundary conditions. To this end, the interested reader is referred to [3].

\section{Evaluation of Effective Elastic Properties}

When limiting our attention to linear elasticity the classical micromechanical models such as the MoriTanaka (MT) method are often preferred to more complex finite element based simulations employing SEPUC. Since the latter approach is adopted in the next section addressing the loading conditions beyond the elastic limits of material phases we compare both methods to support adequacy of the selected RVEs.

The Mori-Tanaka averaging scheme builds upon the knowledge of the shape and orientation of the reinforcements, their material properties and volume fractions. The microstructural details are therefore not fully reflected. The material properties adopted in the presented study for individual phases are listed in Tab. 2 These are derived from experimental measurements, recall Tab. 1] and partly taken from literature [5]. The homogenized properties of the basalt yarn are then summarized in Tab. 3. It is clear that in case of elasticity both analytical and numerical homogenization provide the results which are reasonably close. This also partly supports application of the two computational models for the nonlinear analysis, not quite possible with the MT method. 


\begin{tabular}{lccccc}
\hline Material & $\mathbf{E}$ & $\mathbf{G}$ & $\nu$ & $\mathbf{G}_{f}$ & $\mathbf{S}_{T}$ \\
& {$[\mathbf{G P a}]$} & {$[\mathbf{G P a}]$} & {$[-]$} & {$[\mathbf{N} / \mathbf{m m}]$} & {$[\mathbf{G P a}]$} \\
\hline Basalt fiber - longitudinal direction & 69.68 & 28.10 & 0.24 & - & 2 \\
Basalt fiber - transverse direction & 64.82 & 26.14 & 0.24 & - & 0.5 \\
Pyrolyzed polysiloxane matrix & 58.83 & 23.72 & 0.24 & 0.001 & 0.08 \\
\hline
\end{tabular}

TABLE 2. Considered parameters of individual materials.

\begin{tabular}{lccccccc}
\hline Model type & $\mathbf{E}_{11}$ & $\mathbf{E}_{22}$ & $\mathbf{E}_{33}$ & $\mathbf{G}_{23}$ & $\mathbf{G}_{31}$ & $\mathbf{G}_{12}$ & $\mathbf{c}_{f}$ \\
& {$[\mathbf{G P a}]$} & {$[\mathbf{G P a}]$} & {$[\mathbf{G P a}]$} & {$[\mathbf{G P a}]$} & {$[\mathbf{G P a}]$} & {$[\mathbf{G P a}]$} & {$[-]$} \\
\hline SEPUC-1 - FEM & 62.96 & 62.97 & 65.86 & 26.46 & 26.48 & 23.5 & 0.62 \\
SEPUC-2 - FEM & 62.98 & 62.96 & 65.86 & 26.48 & 26.46 & 23.5 & 0.62 \\
Mori-Tanaka method & 62.97 & 62.97 & 65.88 & 26.47 & 26.47 & 23.5 & 0.62 \\
\hline
\end{tabular}

TABLE 3. Effective elastic moduli.

\section{Damage Modeling at the Level OF YARNS}

Henceforth, we concentrate on the description of damage evolution within a single yarn of the present textile composite. Similar to the previous section, the yarn is being considered as a unidirectional composite (UD) subjected to macroscopically uniform strain. Attention is accorded to transverse direction only. An improved damage evolution model proposed by Fang et al. [6, 7] is applied to receive a more reliable response after the onset of cracking in comparison to, e.g. the direct stiffness reduction method.

Given the orthotropic character of UD composites we associate with each principal direction an independent damage parameter $\omega_{i}, \mathrm{i}=1,2,3$. Additionally, six damage parameters $\mathrm{d}_{1 T}, \mathrm{~d}_{1 C}, \mathrm{~d}_{2 T}, \mathrm{~d}_{2 C}, \mathrm{~d}_{3 T}$ and $\mathrm{d}_{3 C}$ are specified to further distinguish the failure in tension ( $\mathrm{T}$ ) and compression (C). Their evolution is assumed in terms of an equivalent displacement $\chi_{e q}$. The damage parameter $\mathrm{D}$ reflecting a material degradation with the evolution of cracks is expressed in the form

$$
D_{J}=\frac{\chi_{e q}^{J f}\left(\chi_{e q}^{J}-\chi_{e q}^{J i}\right)}{\chi_{e q}^{J}\left(\chi_{e q}^{J f}-\chi_{e q}^{J i}\right)}, \quad J=1 T, 2 T, 3 T .
$$

In Eq. (1), $\chi_{e q}^{J i}$ represents the equivalent displacement at the onset of failure whereas $\chi_{e q}^{J f}$ is the full damage equivalent displacement, see [6, 7] for more details. For both phases, fibers and matrix, the crack initialization is controlled by the simple maximum stress criterion. The corresponding tensile strengths are stored in Tab. 2. Note that compressive failure was not addressed in this study.

\subsection{Numerical ExPeriment}

The two types of SEPUC in Fig. 4 representing the yarn were analyzed to show the influence of the damage parameter evolution on the mesoscopic stressstrain curve. The tensile load was controlled by the increments of the macrostrain in the two main directions perpendicular to the direction of fibers. The first
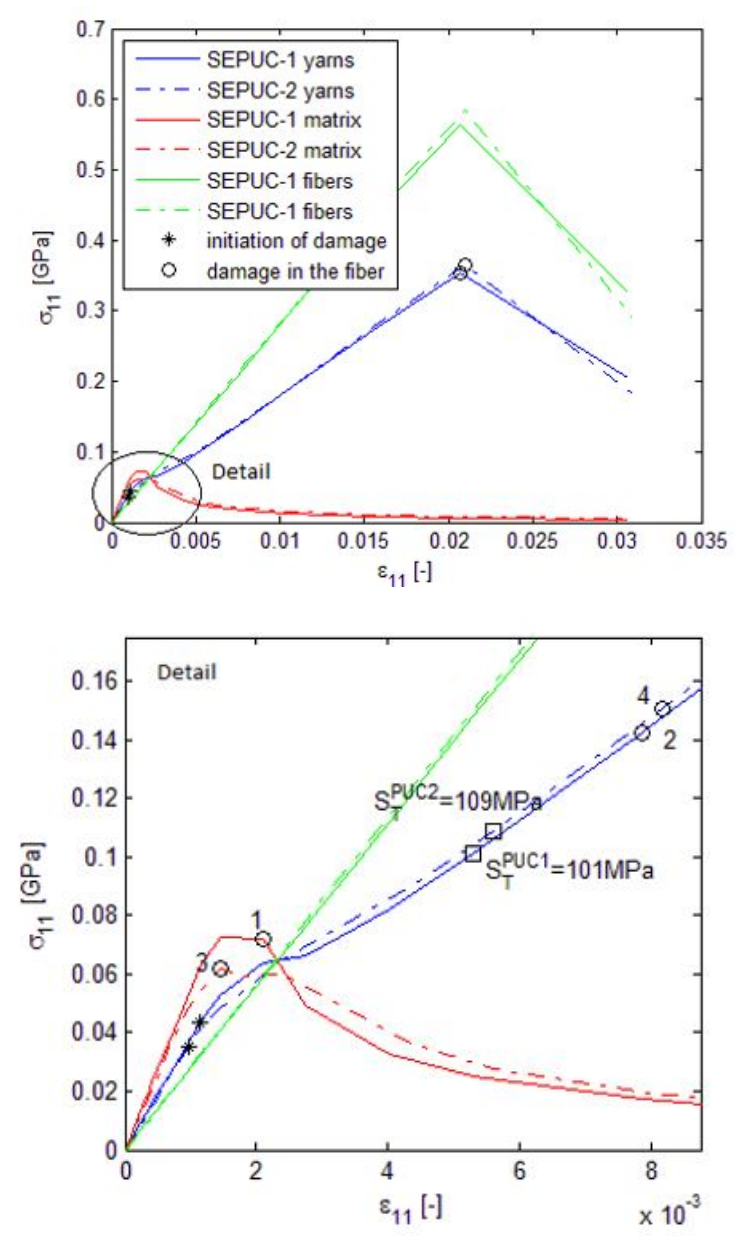

FIGURE 5. Stress-strain curves under the macroscopic tensile strain in the first transverse direction.

main direction corresponds to the $\mathrm{x}$-axis, the second to y-axis, recall Fig. 4 The resulting stress-strain curves are plotted in Figs. 5 and 7

The blown-up parts in Fig. 5 and 7 identify 2 points for both the SEPUC-1 (points 1,2) and SEPUC-2 (points 3,4) for which the evolution of damage parameter, ranging in general from 0 to 1 , is plotted in Fig. 6 and Fig. 8 for both loaded directions. 

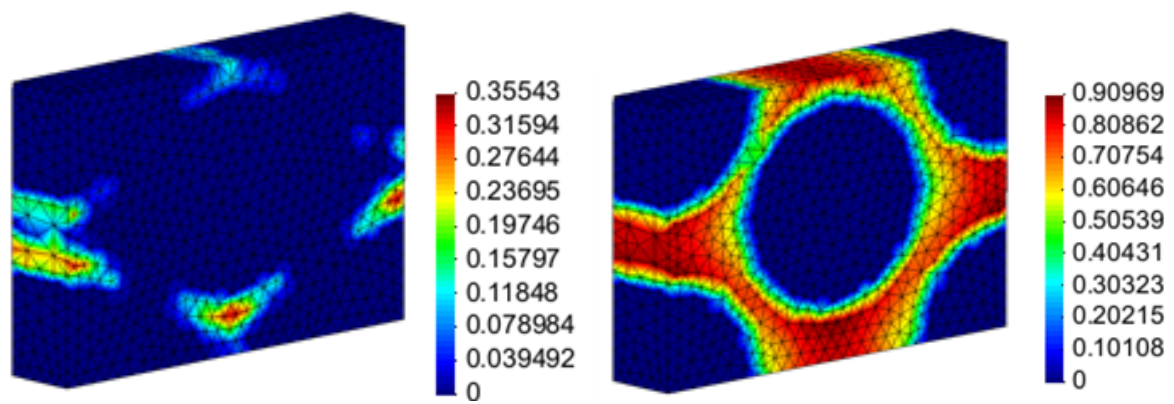

(A) . SEPUC-1 - point 1 and 2
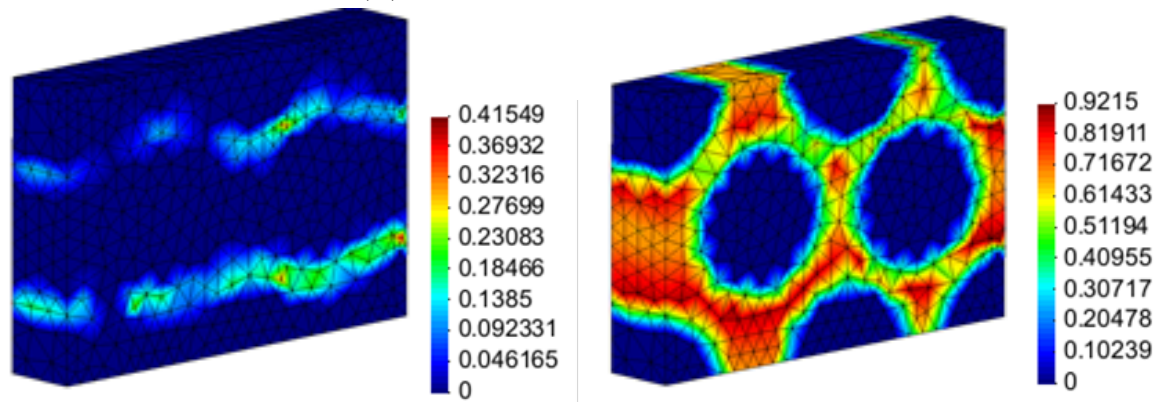

(в) . SEPUC-2 - point 3 and 4

Figure 6. Damage evolution in the matrix corresponding to the marked numbered points in Fig. 5
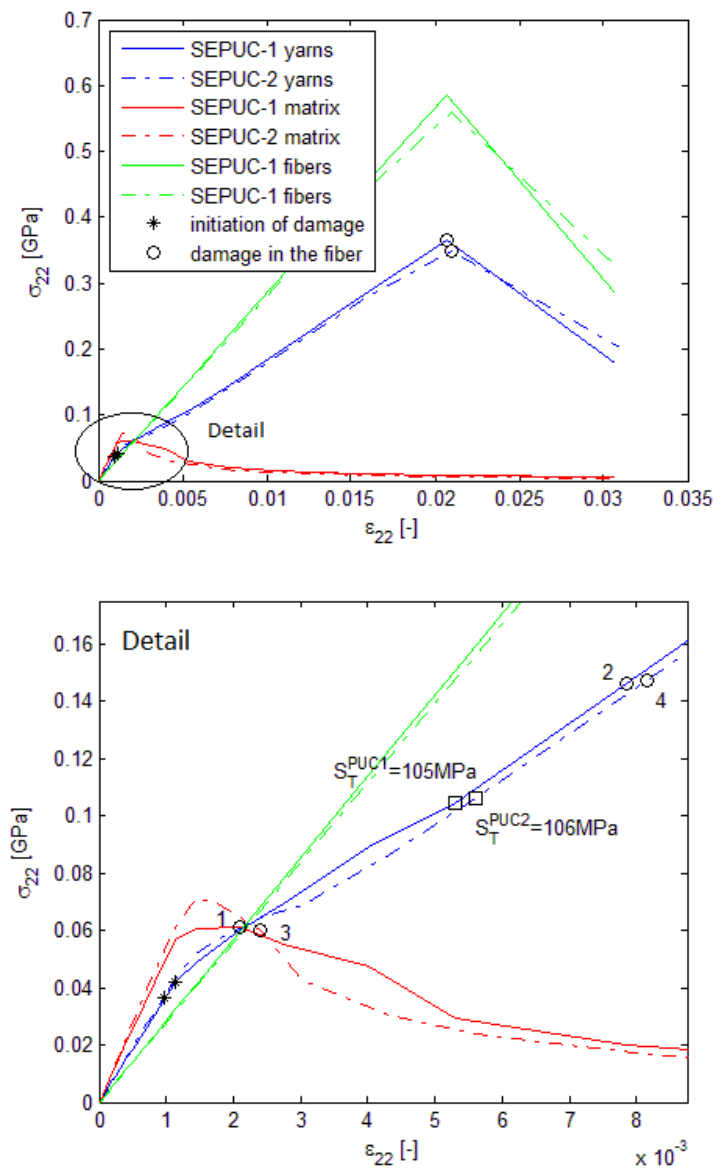

Figure 7. Stress-strain curves under the macroscopic tensile strain in the second transverse direction.
As you can see in Fig. 6 and 8 the damage initiation in the matrix loaded in tension occurs in both directions relatively soon. This corresponds to the macroscopic stress value of about $40 \mathrm{MPa}$. The damage in the matrix phase then progresses extensively before the fiber failure takes place.

The mesoscopic strength parameter $\mathrm{S}_{T}$ can be derived as a value of stress in the stress-strain curve after which the damage parameter starts to rapidly grow. These values are indicated in Fig. 5 and 7 for individual loading directions, respectively. Point out that the hexagonal arrangement of fibers in SEPUC-1 should give an isotropic response. A slight difference between the two directions can therefore be attributed the unstructured (nonuniform) finite element mesh.

\subsection{Summary}

The presented paper is focused on the evaluation of the effective properties of the basalt textile ceramic matrix reinforced composites at the level of yarns with a special attention paid to the determination of the macroscopic tensile strength using computational homogenization. First the homogenized elastic moduli were obtained with the help of the Mori-Tanaka averaging scheme and FEM simulations to support the adequacy of the two selected computational models (SEPUC-1 and SEPUC-2).

The elastic material properties of individual phases obtained from experimental measurement and partly also from literature. Then the virtual experiment on these cells was carried out, the tensile macroscopic strain was applied to these cells aiming at the determination of the macroscopic tensile strength. Apart from that, the mesoscopic fracture energy can also be 

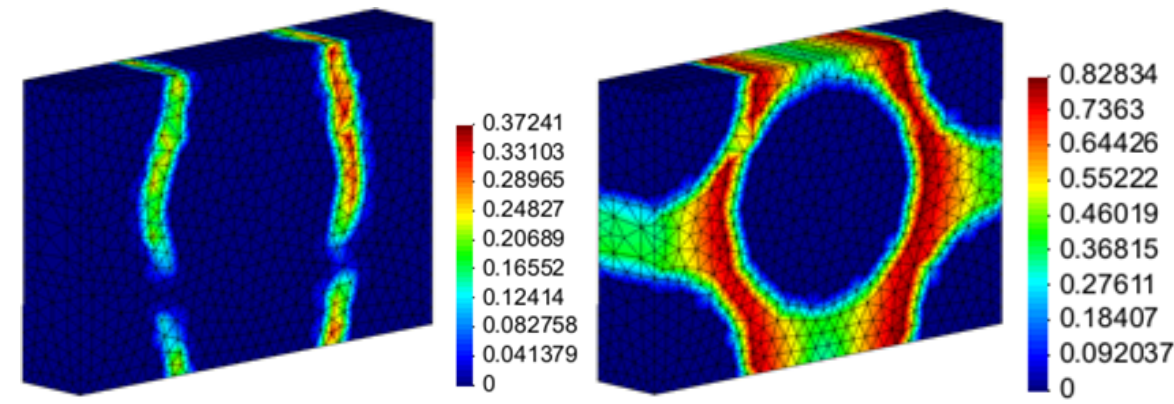

(A) . SEPUC-1 - point 1 and 2

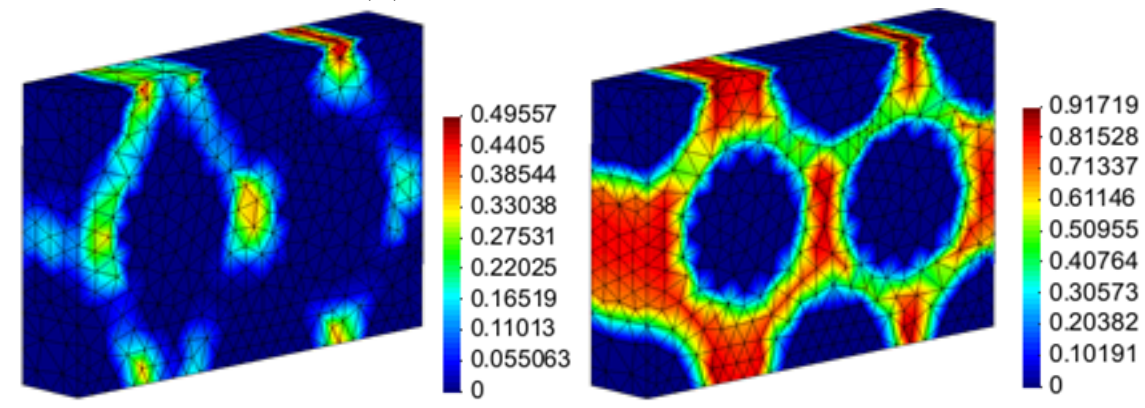

(в) . SEPUC-2 - point 3 and 4

FiguRE 8. Damage evolution in the matrix corresponding to the marked numbered points in Fig. 7

determined from the homogenized stress-strain curves, recall blue lines in Figs. 5 and 7.

These parameters will then enter the numerical homogenization step on mesoscale adopting more complex unit cells as the one schematically plotted in Fig. 4. Point out, however, that the fracture properties of both the matrix and fiber were selected in the present study ad hoc and they still need to be properly measured. To that end, nanoindentation is again expected as a suitable method of attack.

\section{LIST OF SYMBOLS}

$R_{q}$ Root mean square roughness [nm]

$F \quad$ Applied force $[\mu \mathrm{N}]$

$\nu$ Poisson ratio $[-]$

$E_{r}$ Reduced modulus [GPa]

$E$ Young's modulus [GPa]

$H$ Hardness [GPa]

$h_{c}$ Contact depth [nm]

$G$ Shear modulus [GPa]

$G_{f}$ Fracture energy [N/mm]

$S_{T}$ Critical strength in tension [GPa]

$E_{i j}$ Homogenized elastic modulus [GPa]

$G_{i j}$ Homogenized shear modulus [GPa]

$D_{J}$ Damage parameter [-]

$\chi_{e q}$ Equivalent displacement [m]

$\sigma_{i j}$ Applied stress [GPa]

$\epsilon_{i j}$ Resulting strain [-]

\section{ACKNOWLEDGEMENTS}

The financial support provided by the SGS projects with the application registered under the No. OHK1-014/17 and OHK1-031/17 is gratefully acknowledged.

\section{REFERENCES}

[1] H. Hertz. On the contact of rigid elastic solids. chap. 6. Hertz's Miscellaneous Papers, London, 1896.

[2] W. Oliver, G. Pharr. Measurement of hardness and elastic modulus by instrumented indentation: Advances in understanding and refinements to methodology. Journal of Material Research 19(1):3-20, 2004. DOI:10.1557/jmr.2004.0002.

[3] M. Sejnoha, J. Zeman. Micromechanics in practice. WIT Press, Boston, 2013.

[4] J. Zeman, M. Šejnoha. From random microstructures to representative volume elements. Modelling and Simulation in Materials Science and Engineering 15(4):325-335, 2007. DOI:10.1088/0965-0393/15/4/S01.

[5] M. Černý, P. Glogar, Z. Sucharda. Mechanical properties of basalt fiber reinforced composites prepared by partial pyrolysis of a polymer precursor. Journal of Composite Materials 43(9):1109-1120, 2009. DOI:10.1177/0021998308097732

[6] G. Fang, J. Liang, Q. Lu. Investigation on the compressive properties of the three dimensional four-directional braided composites. Composite Structures 93(2):392-405, 2011. DOI:10.1016/j.compstruct.2010.09.002

[7] G. Fang, J. Liang, B. Wang. Progressive damage and nonlinear analysis of 3d four-directional braided composites under unidirectional tension. Composite Structures 89(1):126-133, 2009. DOI:10.1016/j.compstruct.2008.07.016 